\title{
Enhancing the feature-based 3D deformable face recognition using hybrid PCA-NN
}

\author{
Cahyo Darujati ${ }^{1}$, Supeno Mardi Susiki Nugroho², Deny Kurniawan ${ }^{3}$, Mochamad Hariadi ${ }^{4}$ \\ ${ }^{1,2}$ Department of Electrical Engineering, Faculty of Intelligent Electrical and Informatics Technology (ELECTICS), \\ Institut Teknologi Sepuluh Nopember (ITS) Surabaya, Indonesia \\ ${ }^{3}$ Nijmegen School of Management, Radboud Universiteit, Netherland \\ ${ }^{1}$ Faculty of Computer Science, Universitas Narotama, Surabaya, Indonesia \\ ${ }^{4}$ Department of Computer Engineering, Faculty of Intelligent Electrical and Informatics Technology (ELECTICS), \\ Institut Teknologi Sepuluh Nopember (ITS) Surabaya, Indonesia
}

\begin{tabular}{|c|c|}
\hline Article Info & ABSTRACT \\
\hline Article history: & \multirow{10}{*}{$\begin{array}{l}\text { Facial recognition is one of the most important advancements in image } \\
\text { processing. An important job is to build an automated framework with the } \\
\text { same human capacity's for recognizing face. The face is a complex 3D } \\
\text { graphical model, and constructing a computational model is a challenging } \\
\text { task. This paper aims at a facial detection technique focused on the coding } \\
\text { and decoding of the facial feature object theory approach to data. One of the } \\
\text { most natural and common principal component analysis (PCA) method. This } \\
\text { approach transforms the face features into a minimal set of basic attributes, } \\
\text { peculiarities, which are the critical components of the original learning image } \\
\text { collection (or the training package). The proposed technique is a combination } \\
\text { of the PCA system and the identification of components using the neural } \\
\text { network (NN) feed-forward propagation method. This experiment proves that } \\
\text { recognition of deformed 3D face is doable. By taking into account almost all } \\
\text { forms of feature extraction and engineering, the NN yields a recognition } \\
\text { score of } 95 \% \text {. }\end{array}$} \\
\hline Received Oct 20, 2020 & \\
\hline Revised Jan 6, 2021 & \\
\hline Accepted Jan 17, 2021 & \\
\hline Keywords: & \\
\hline 3D deformable model & \\
\hline Facial recognition & \\
\hline Feature engineering & \\
\hline Neural network & \\
\hline Principal component analysis & \\
\hline
\end{tabular}

This is an open access article under the CC BY-SA license.

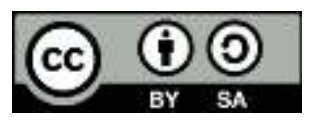

\section{Corresponding Author:}

Mochamad Hariadi

Department of Computer Engineering

Institut Teknologi Sepuluh Nopember (ITS)

Gedung B, C \& AJ Kampus ITS, Sukolilo, Kota Surabaya, Jawa Timur 60111, Indonesia

Email: mochar@ee.its.ac.id

\section{INTRODUCTION}

3D face modeling is a challenging subject in computer graphics and computer vision [1]. More so than 2D face models, 3D face models can genuinely convey face deformation and show variability through greater details [2-3]. With these in mind, 3D face models have been applied to a range of applications involving movies, 3D animation, and telecommunications. It is quite challenging to develop a suitable program to recognize a face digitally, because humans' faces are complex and in every aspect is different. Therefore, in this digital world, creating such a system, which may include prior methods used to identify the eyes, is an arduous task. The foremost step comprises extracting the correct features from the facial expressions for object recognition [4]. A major challenge is how facial features can be quantified so that a machine can identify the eyes $[5,6]$. The study done by various scientists during recent years reveals that human beings use certain facial features to recognize eyes.

Bledsoe, Chan, and Bisson identified human faces' identification process with a computer-aided program [7]. Much of the study worked on defining individual features, such as eyebrows, pupils, nose, and 
lips. By giving their attention to representing the entire face, the development of facial recognition using statistical methods has evolved [8,9]. In general, facial recognition approaches can be divided into three main groups of the recognition process, e.g., based on function, look, and template.

One of the key modules in the face recognition system is the feature extraction function, which has a major impact on the system's overall performance. Over the past decades, different feature extractors, descriptors, encoders, and decoders have been proposed for 3D face recognition [10-12]. While many literature reviews have been conducted on 3D face recognition algorithms, only a few studies have been conducted on said feature engineering methods, let alone the $3 \mathrm{D}$ deformable model. They have a crucial role in resolving degradation situations, such as facial expression changes, occlusions, aging and declining physical attributes [13].

There has been a lot of work in facial recognition in the last few years, and they have found progress in actual use. These algorithms can be classified into two dimensional (2D) and three dimensional (3D) approaches. The traditional 2D approaches are primarily divided into six algorithms: PCA, LDA, ICA, SVM, NN, and HMM $[14,15]$. Turk and Pentland suggested the most popular technique to analyze the principal component, or famously known as principle component analysis (PCA). It is mostly used to reduce dimensionality and extract functionality in most pattern recognition applications $[16,17]$. A modular multiple correspondence analysis (MCA), in an FPGA environment, is a scalable and robust architecture for the realtime face recognition framework [18]. Modular PCA has been demonstrated to enhance facial recognition performance when facial pictures have different expression and illumination [19].

The process in facial recognition techniques is generally divided into several stages, namely the processing, segmentation, feature extraction, and the identification process, with the most popular algorithms using PCA [15]. With the PCA method, the image size is to be trained and tested by the same size. Therefore, the normalization process is done by aligning the eye and mouth area. All images in the training process will be stored in a T-matrix in vector rows, and these rows represent a source of processed images [20]. Then, the average value will be subtracted from each source image on the T-matrix. The next step is to calculate the eigenvector value and the eigenvalue of the covariance matrix S.The eigenvector is a value resulting from a reduction in size by removing some unnecessary information and splitting the structure of the face pattern into components that are not related to the shape of the face itself.

In this work, we tried to take advantage of PCA by reducing the eigenvector value. Although PCA minimizes the number of 3D face features, it doesn't necessarily mean it will negatively impact the accuracy result or computation time. Previous works mentioned computation time and overall accuracy rate improvement. Experiments were carried out using the UoY 3D face dataset [21]. Using our proposed approach, the overall distance value of $3 \mathrm{D}$ face features has not changed, and the FAR value can be adequately reduced [22].

The rest of this paper is organized as follows: Section 1 briefly described the PCA method (eigenfaces) for the face recognition technique, with several supporting literature review. Section 2 explained the proposed methodology that we used in this face recognition. Finally, in Section 3, we present our experiment result and discussion, then conclude this work in Section 4.

\section{RESEARCH METHOD}

The proposed methodology experiments with deformed 3D facial images, focusing on local and global face features. This process collects, encodes, and then compares the relevant information from a facial image to a facial template database and then proceed to classification using NN. The method's main advantage is the capture of features such as closed and open eyes timing, different facial expressions, and faces with glasses.

\subsection{Pre-processing step of human $3 D$ face dataset}

This experiment's first step is data pre-processing, which includes the normalization of image width, histogram equalization, and transformation to greyscale. Our system automatically reduces face images to multiple $X$ pixels and $Y$ pixels to increase face image quality and face image strength (using histogram equalization). Images were later stored in a facial archive in the system. Stored images were divided on the scale 60:40 from the facial image total percentage for the training set and testing set, respectively. The traditional training and testing set are done using our built NN.

The next important step of the facial recognition process is extracting facial features. Extraction is carried out to find essential parts of the human face. Some of the facial features are the contours of all the critical areas around the eyes, nose, and mouth. The process begins by creating a feature line model used to draw the rotational shape and part of the region's extracted feature points. 


\subsection{Eigenface descriptor}

PCA is a common technique for alleviating high dimensional vectors in feature dimensions, be it text's, voice's, and most definitely in faces'. In some cases, this may result in a significant reduction in computing time with little or no impact on the recognition rate. It may also be used to evaluate the orthogonal base set for redundant functions, such as raw pixels in small-windowed images.

The linear combination form of Eigenfaces values can represent many face images accurately. Only the face with the best $M$ value can approach the original face data by its most immense value. The best value $M$ of Eigenfaces includes each subspace (that is $M$-dimensional). As stated in the previous chapter, the PCA algorithm suggested by Kirby and Sirovich can be used to measure the value of Eigenfaces. This algorithm involves calculating the cumulative difference for each measured face and the mean face space value. Pseudocode of PCA Eigenface approach as shown in Table 1.

Table 1. Pseudocode of PCA Eigenface Approach

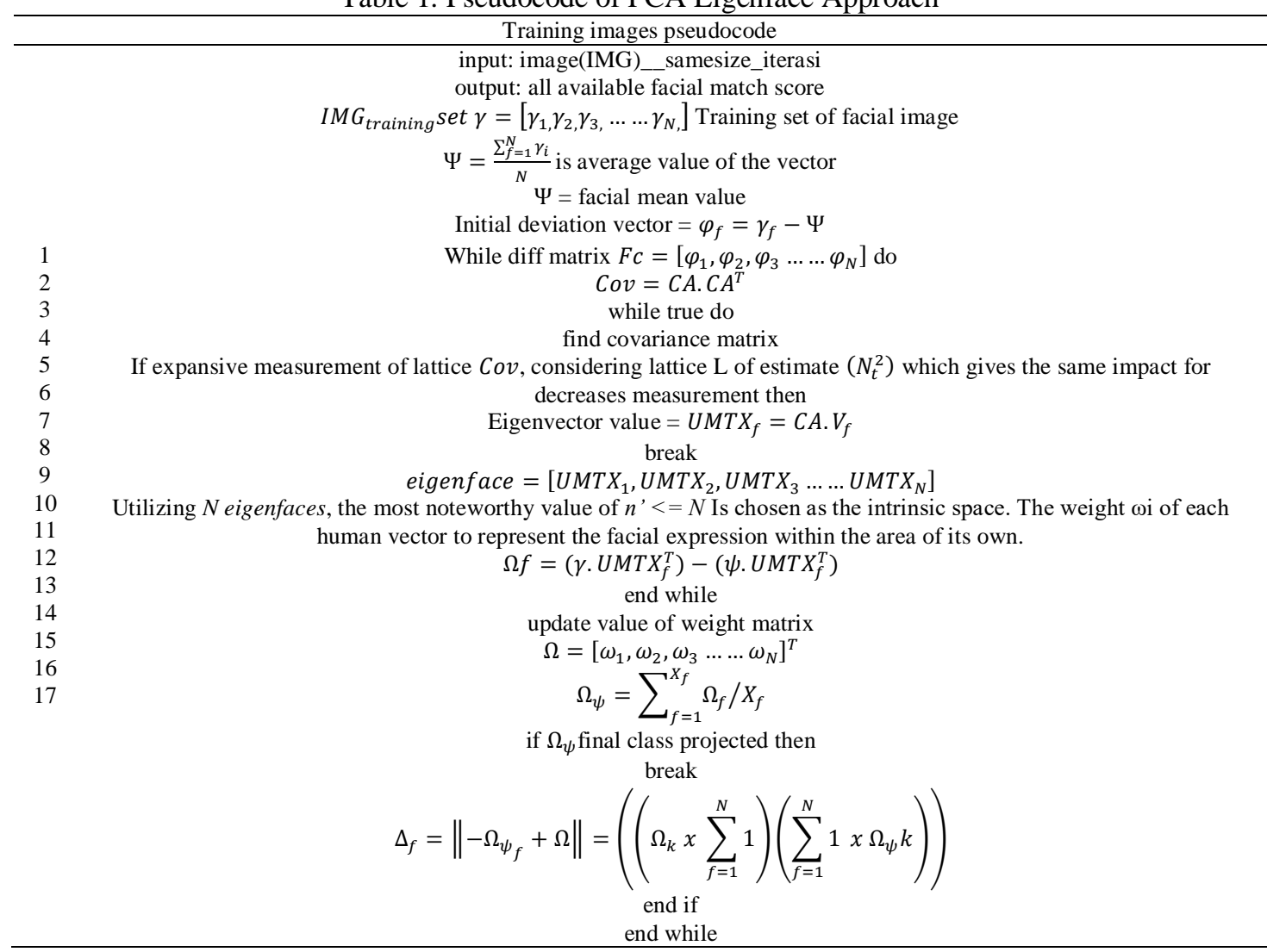

The next step is to calculate the data set into a matrix with the covariance difference $C$. This covariance matrix derived from the data set shows the relationship between the matrix sets. Based on the PCA numerical method's technical steps, the number of vectors of the covariance matrix value makes it possible to reduce the total amount of pixels in the face image from $N$ to the total number of pictures in the training data. The whole face image is normalized by changing each face image grid's value into a vector value of a comparable face image. All dataset was defined as a set of face image vectors with $I M G_{\text {training }}$ set $\gamma$.

\subsection{Neural networks for training}

The sample used in this analysis includes more than a collection of human face photographs with various conditions. The subject can be seen in each image by the size of $N \times Y$ pixels with 256 degrees of gray-ish level. For some subjects, pictures are taken at different times, with the exposure variations, the facial expressions. The initial image was resized to $40 \times 40$, and the total dimension magnitude of the display space is 1,600. The value $M$ of Eigenfaces is calculated using the PCA algorithm. The initial experiment from the number of human faces in each face space was varied to determine the picture's number of facial descriptors. The $\mathrm{NN}$ in our experiment is used to train the network by the number of the face descriptor [23]. 
The input comes from the same person's face data, which is used during the learning process as a positive value for individual networks; on the contrary, it is used on a different network for negative examplesmeanwhile, Figure 1 shown the NN learning schematic diagram.

In Figure 2, the output classes are equal to the total subjects in the dataset. A total of $60 \%$ of the face images dataset are used for the NN training process, then NN is tested, and the weight value is updated. The trained network will later be used for comprehensive facial recognition purposes. In other words, a set of face images is used differently in both training and testing to serve its purpose well. The entire value of the global performance results will appear for the whole dataset.

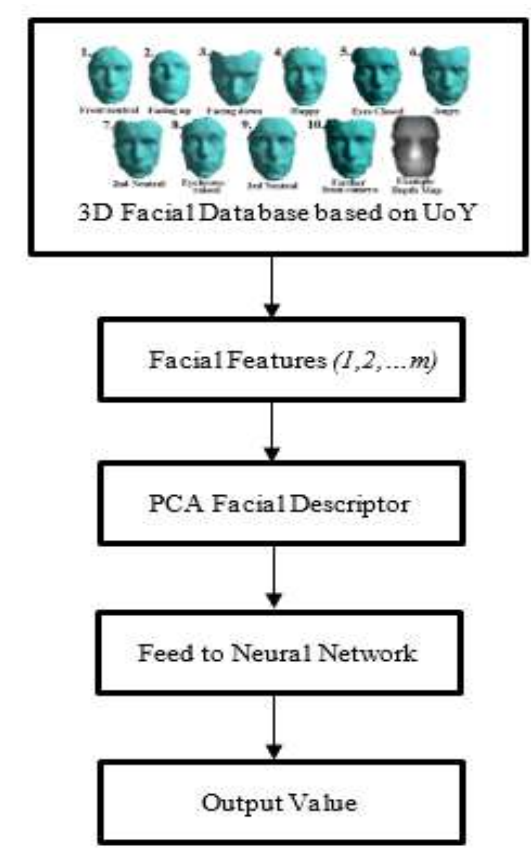

Figure 1. Facial Recognition Steps using Neural Network

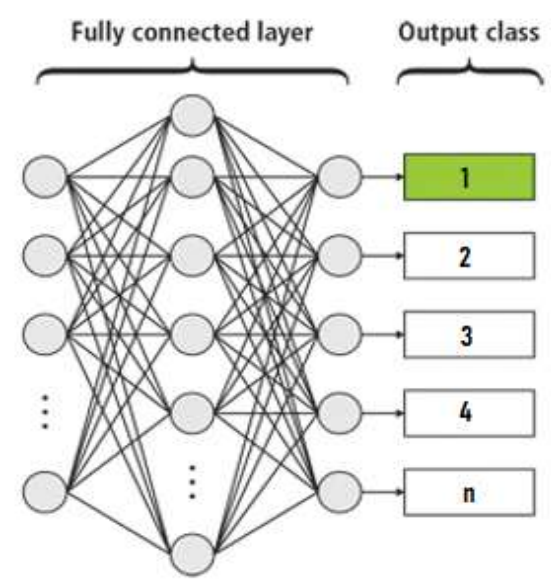

Figure 2. The Architecture of Our Neural Network

\section{RESULTS AND DISCUSSION}

In this experiment, we divided the data composition scale into 60:40, respectively for training and testing stage. In the training stage, the number of images is increased by one step, which has initial 20 images in set. These results indicate that using initial 20 images in the training set for each subjects' face meets a value of $100 \%$, which is expressed in a chart in Figure 3. 


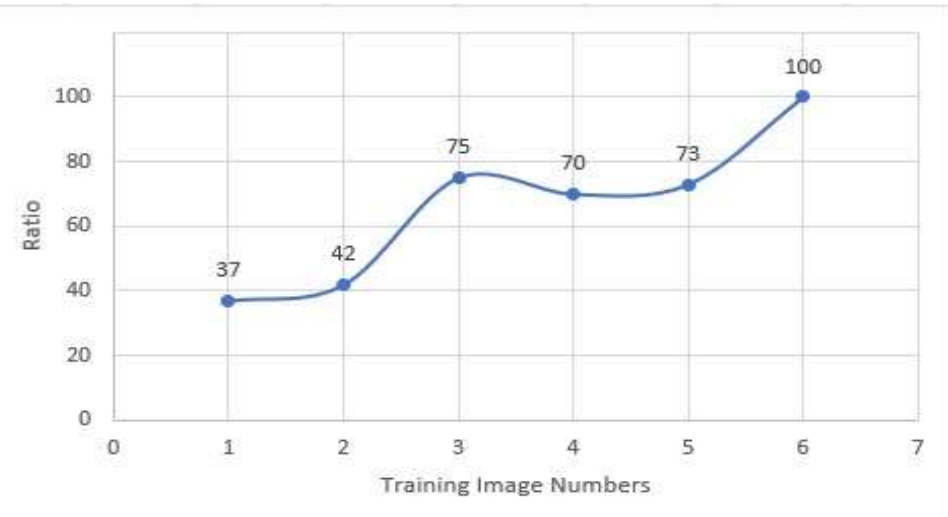

Figure 3. Training image number per ratio chart

Later, we carried out the testing process on 40 facial data, each with ten images in the training database. The threshold value is based on the Euclidean distance with the minimum value. The Euclidean distance is the distance between the projected range value of the test image and all images' projection at the training stage. The test image stage has a minimum value distance with the appropriate image in the training stage. To simplify the threshold, we divided it by $1.0 \mathrm{E}+18$ ); this value is used to determine the equilibrium threshold value, in which the result is shown in Figure 4. The curve's value indicates the result as an equilibrium value that appears at the same threshold as $4.75+$ E18. However, to obtain a much better accuracy value, we chose the threshold value to be $4.50+\mathrm{E} 18$, which is close to zero. We included over 180 eigenvector values and used the time measure for each recognition process. For the recognition process, the period ranges from 1.3 to 1.7 seconds. Figure 3 shows the trade-off value between FAR and FRR that occurred in the second-stage experiment. The result in Figure 4 shows the total value of the FRR and FAR in comparison.

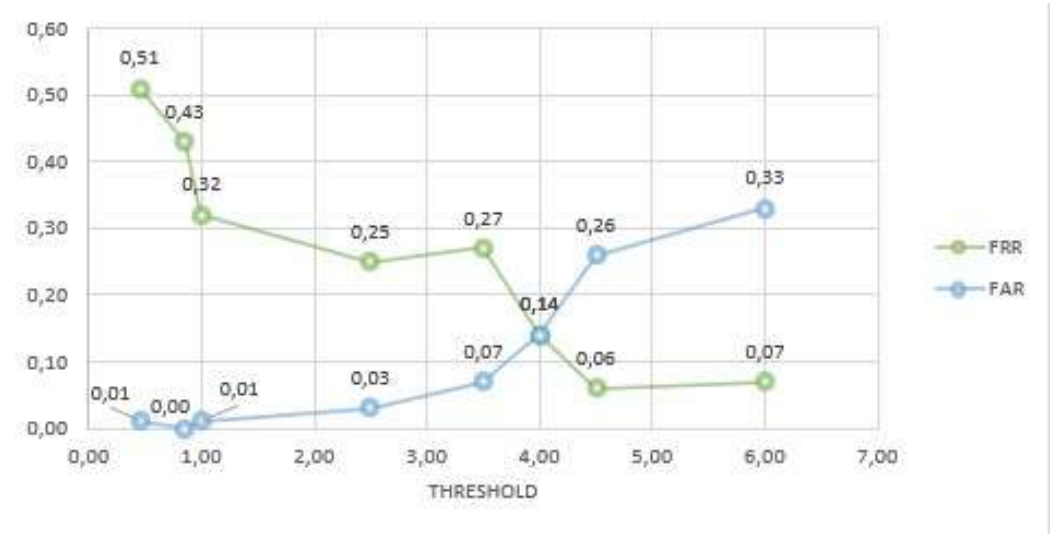

Figure 4. FRR and FAR chart in comparison

A sorting process of PCA carries out the Eigenvalues in question; if the value is less than the predetermined threshold, an elimination process will be carried out. In the second-stage experiment, the findings in the form of eigenvector values are in the range $-1.778 \mathrm{E}-005$ to $3.758 \mathrm{E}+007$. The results of this experiment indicate a significant reduction in computation time. By applying the PCA algorithm, the computation time spent was in the range of 0.7 seconds to 1.1 seconds while maintaining the same threshold value at zero FAR. Although using the same method (PCA), our result proved to be superior than the ones that have been worked in [24], which has computation time above $300 \mathrm{~ms}$. The one work in [25] even yield $\sim 11$ seconds. Without PCA, the computation time can yield about one to five hours, as stated in [26]. 


\section{CONCLUSION}

This paper presents an engineering technique that combines two methods to improve the accuracy value of the three-dimensional facial detection process using PCA and NN. The accuracy value obtained provides a better recognition rate than other techniques, with the computation time result to be at least faster than other works (ranging from 0.7 to 1.1 seconds). Moreover, it is worth to be noted that we have a high variation value in the Eigenfaces for the entire dataset. Using the Eigenfaces method, the value obtained is very sensitive to the head orientation's shape or position. In some cases, this happens because there are differences in the dataset whose orientation shape of a large head. To alleviate this problem, we chose to employ PCA. Future research aims to establish a particular framework and carry out the initial process for more detailed feature extraction. The NN has been proven to be able to complete face detection correctly; this can be considered in the future.

\section{ACKNOWLEDGEMENTS}

We would like to thank the Ministry of Research and Technology, Republic of Indonesia/BRIN for funding this research the under BPPDN grant schema.

\section{REFERENCES}

[1] J. Booth, E. Antonakos, S. Ploumpis, G. Trigeorgis, Y. Panagakis, and S. Zafeiriou, "3d face morphable models," in 2017 IEEE Conference on Computer Vision and Pattern Recognition (CVPR), pp. 5464-5473, 2017.

[2] Z. H. Choudhury, "Biometrics security based on face recognition," 2020.

[3] H. Dai, N. Pears, P. Huber, and W. A. P. Smith, "3D Morphable Models: The Face, Ear and Head," in 3D Imaging, Analysis and Applications, Springer, pp. 463-512, 2020.

[4] Y. Zhang and Z. Wang, "Gait recognition using principal component analysis," Int. J. Adv. Comput. Technol., vol. 4, no. 22, pp. 600-607, 2012, doi: 10.4156/ijact.vol4.issue22.68.

[5] H. Admoni and B. Scassellati, "Social Eye Gaze in Human-Robot Interaction: A Review," J. Human-Robot Interact., vol. 6, no. 1, p. 25, Mar. 2017, doi: 10.5898/JHRI.6.1.Admoni.

[6] Y. Chen, J. Qian, J. Yang, and Z. Jin, "Face alignment with Cascaded Bidirectional LSTM Neural Networks," Proc. - Int. Conf. Pattern Recognit., vol. 0, no. 2, pp. 313-318, 2016, doi: 10.1109/ICPR.2016.7899652.

[7] I. Masi, Y. Wu, T. Hassner, and P. Natarajan, "Deep Face Recognition: A Survey," in 2018 31st SIBGRAPI Conference on Graphics, Patterns and Images (SIBGRAPI), pp. 471-478, Oct. 2018, doi: 10.1109/SIBGRAPI.2018.00067.

[8] J. Matai, A. Irturk, and R. Kastner, "Design and Implementation of an FPGA-based Real-Time Face Recognition System," in 2011 IEEE 19th Annual International Symposium on Field-Programmable Custom Computing Machines, pp. 97-100, 2011.

[9] Y. A. Jasim, A. A. Al-Ani, and L. A. Al-Ani, "Iris recognition using principal component analysis," Proc. - 2018 1st Annu. Int. Conf. Inf. Sci. AiCIS 2018, vol. 5, no. 5, pp. 89-95, 2019, doi: 10.1109/AiCIS.2018.00028.

[10] Y. Li, W. Zheng, Z. Cui, and T. Zhang, "Face recognition based on recurrent regression neural network," Neurocomputing, vol. 297, pp. 50-58, 2018.

[11] M. O. Oloyede, G. P. Hancke, and H. C. Myburgh, "A review on face recognition systems: recent approaches and challenges," Multimed. Tools Appl., vol. 79, no. 37, pp. 27891-27922, 2020.

[12] Q. Duan and L. Zhang, "Look More Into Occlusion: Realistic Face Frontalization and Recognition With BoostGAN," IEEE Trans. Neural Networks Learn. Syst., 2020.

[13] Z. Cheng, X. Zhu, and S. Gong, "Low-resolution face recognition," in Asian Conference on Computer Vision, pp. 605-621, 2018.

[14] Z. B. Lahaw, D. Essaidani, and H. Seddik, "Robust Face Recognition Approaches Using PCA, ICA, LDA Based on DWT, and SVM Algorithms," in 2018 41st International Conference on Telecommunications and Signal Processing (TSP), pp. 1-5, 2018.

[15] R. Sutar, S. Rokadia, and A. Shah, "A survey on face recognition technologies and techniques," in International Journal of Technology and Computing (IJTC), vol. 2, no. 7, July, 2016, 2016.

[16] M. Abdullah, M. Wazzan, and S. Bo-Saeed, "Optimizing face recognition using PCA," arXiv Prepr. arXiv1206.1515, 2012.

[17] H. M. Ebied, "Feature extraction using PCA and Kernel-PCA for face recognition," 2012.

[18] S. Pouyanfar, Y. Tao, H. Tian, S.-C. Chen, and M.-L. Shyu, "Multimodal deep learning based on multiple correspondence analysis for disaster management," World Wide Web, vol. 22, no. 5, pp. 1893-1911, 2019.

[19] P. Peng, P. Alencar, and D. Cowan, "A Software Framework for PCA-Based Face Recognition," Proc. - 2016 IEEE Int. Conf. Softw. Sci. Technol. Eng. SwSTE 2016, pp. 7-16, 2016, doi: 10.1109/SWSTE.2016.11.

[20] Y. Yang, G. Zhang, D. Katabi, and Z. Xu, "Me-net: Towards effective adversarial robustness with matrix estimation," arXiv Prepr. arXiv1905.11971, 2019.

[21] N. Pears and A. Mian, "3D Face Recognition," in 3D Imaging, Analysis and Applications, Springer, pp. 569-630, 2020.

[22] S. N. Sujay, H. S. M. Reddy, and J. Ravi, "Face recognition using extended LBP features and multilevel SVM 
classifier," in 2017 International Conference on Electrical, Electronics, Communication, Computer, and Optimization Techniques (ICEECCOT), pp. 1-4, 2017.

[23] V.-E. Neagoe and I.-F. Iatan, "Face recognition using a fuzzy-Gaussian neural network," in Proceedings First IEEE International Conference on Cognitive Informatics, pp. 361-368, doi: 10.1109/COGINF.2002.1039318.

[24] S. Xue, "Face Database Security Information Verification Based on Recognition Technology," IJ Netw. Secur., vol. 21, no. 4, pp. 601-606, 2019.

[25] A. L. Ramadhani, P. Musa, and E. P. Wibowo, "Human face recognition application using pca and eigenface approach," in 2017 Second International Conference on Informatics and Computing (ICIC), pp. 1-5, Nov. 2017, doi: 10.1109/IAC.2017.8280652.

[26] F. M. A. Azis, M. Nasrun, C. Setianingsih, and M. A. Murti, "Face recognition in night day using method eigenface," in 2018 International Conference on Signals and Systems (ICSigSys), pp. 103-108, May 2018, doi: 10.1109/ICSIGSYS.2018.8372646.

\section{BIOGRAPHIES OF AUTHORS}

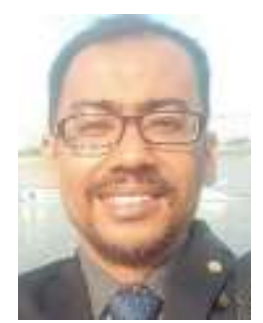

Cahyo Darujati received a bachelor in 2004 and master degree in 2010 respectively from Electrical Engineering Department, Institut Teknologi Sepuluh Nopember, Surabaya, Indonesia. $\mathrm{He}$ is a Professional Lecturer Faculty of Computer Science, Narotama University, Surabaya, Indonesia. His research interest includes computer vision, image processing, and video processing. He is IAENG member.

E-mail: cahyo11@mhs.ee.its.ac.id

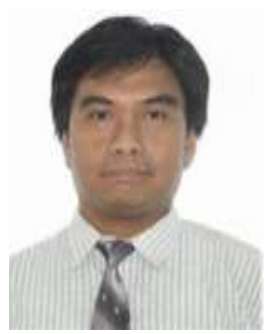

Supeno Mardi Susiki Nugroho graduated from Electrical Engineering Institut Teknologi Sepuluh Nopember (ITS), Surabaya, Indonesia for his bachelor in 1995 and received master and doctoral degree in 2003 and 2013 respectively from Electrical Engineering of Institut Teknologi Sepuluh Nopember (ITS), Surabaya, Indonesia. He is currently a lecturer of Electrical Engineering Department ITS. His research interest includes image processing, computer network, and Artificial Intelligence.

E-mail: mardi@ee.its.ac.id

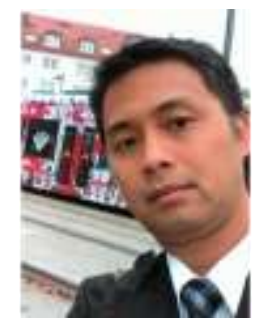

Deny Kurniawan received a bachelor in 2004 from Electrical Engineering Department, Institut Teknologi Sepuluh Nopember, (ITS) Surabaya, Indonesia and received Master Degree in 2013 from Computer Science, University of Indonesia. At the moment $\mathrm{He}$ is a $\mathrm{PhD}$ candidate from Nijmegen Management School, Radboud Universiteit, Netherlands. His research interest includes Higher Education Management and Multi-criteria Decision Analysis. He is a Head Division of Institutional Development, under Directorate General of Higher Education, Ministry of Education and Culture.

E-mail: dkurniawan@kemdikbud.go.id

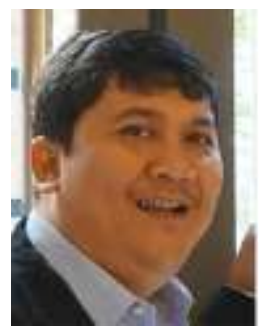

Mochamad Hariadi received the B.E.degree in Electrical Engineering Department of from Institut Teknologi Sepuluh Nopember, Surabaya, Indonesia, in 1995. He received both M.Sc. and Ph.D. degrees in Graduate School of Information Science Tohoku University Japan, in 2003 and 2006 respectively. Currently, he is a staff of the Electrical Engineering Department of from Institut Teknologi Sepuluh Nopember, Surabaya, Indonesia. He is the project leader in joint research with PREDICT JICA project in Japan.

E-mail: mochar@ee.its.ac.id 